\title{
Improvement of QDaedalus measurements with continuous detection of environmental parameters
}

\author{
Lajos Völgyesi $^{1}$ (D) . Gyula Tóth ${ }^{1}$
}

Received: 2 June 2021 / Accepted: 11 August 2021 / Published online: 5 September 2021

(c) The Author(s) 2021

\begin{abstract}
QDaedalus is an automated, computer-controlled astro-geodetic measurement system. Astronomical deflections of the vertical measured by the QDaedalus system are significantly influenced by atmospheric refraction. Therefore, the measuring system was further improved by recording the environmental parameters influencing the refraction (air pressure, temperature, humidity) with accurate and high time resolution. In addition to meteorological parameters, refraction also depends on the spectrum of the stars. Both the continuously measured meteorological parameters and the color of the stars were taken into account in the calculation of the refraction. To control the method, we used the deflection of the vertical values of the Pistahegy point in the southern part of Budapest which were determined over 7 years during 260 night measurements. The corrected measurements fit within $0.01 "$ with the average value of previous Pistahegy measurements. The standard deviation of the differences due to the corrections, however, may reach 0.015 " for the DOV components.
\end{abstract}

Keywords QDaedalus · Astro-geodetic measurement · Deflection of the vertical · Atmospheric refraction $\cdot$ Star spectra $\cdot$ Robust inversion

\section{Introduction}

Gravity as a vector also has length and direction. The length of the vector can be measured by different absolute and relative gravity methods. Direction of gravity (the vertical deflection) can be measured by astrogeodetic methods. Accurate determination of the direction of the gravity vector is an important task in geodesy and for certain engineering projects, but these measurements can also be used in geophysics too.

Classical determination of vertical deflection is a very lengthy and costly job. Measurements are performed based on a pre-prepared star program. Night fieldwork, which

Lajos Völgyesi

volgyesi.lajos@emk.bme.hu

Gyula Tóth

toth.gyula@emk.bme.hu

1 Department of Geodesy and Surveying, Budapest University of Technology and Economics, Mû́egyetem rkp. 3, 1111 Budapest, Hungary 
may last for several weeks, requires serious infrastructure (transport, power supply, service staff, etc.). A suitable pillar must be built with a special observation tent for the measurements. So far, due to the rather lengthy and expensive method of astronomical position determination, such measurements have been performed at very few points, so e.g. currently only 138 points are available in Hungary, which corresponds to an average point distance of about $33 \mathrm{~km}$.

Determining the vertical deflection at a single point by astronomical position determination-including preparations, measurements, and calculations-required several months of work by many professionals. Accuracy of this measurement is about 0.2 arc second. The use of the QDaedalus system is a huge step forward in determining deflection of the vertical (DOV) as a single observer can determine these values not more than 20-30 min with greater accuracy.

The most problematic source of astrodeodetic measurements is atmospheric refraction. The magnitude of refraction depends primarily on meteorological parameters, which vary significantly in space and time. For the precise determination of the vertical deflection, a very long sequence was measured at the point Pistahegy. In the last 7 years we performed 260 measurements taken at this point in different seasons, in the most diverse meteorological, temperature and refraction conditions. In previous measurements, meteorological parameters were determined only at the beginning and end of the measurements, but more recently we have switched to high-precision recording of barometric pressure, temperature, and humidity every $10 \mathrm{~s}$. This made it possible to continuously determine the refraction and take its effect into account during the entire measurement time.

\section{The QDaedalus system}

The QDaedalus system is a computer-controlled, GNSS-assisted automatic measuring system, which can be used mainly for high-precision determination of the local vertical direction (deflection of the vertical=DOV) (Bürki et al., 2010; Hirt et al. 2010; Hauk et al. 2016; Tóth and Völgyesi 2016).

The N-S and E-W component $\xi$ and $\eta$ can be computed by.

$$
\begin{gathered}
\xi=\Phi-\varphi, \\
\eta=(\Lambda-\lambda) \cos \varphi
\end{gathered}
$$

where $\varphi, \lambda$ are geodetic latitude and longitude on WGS84 ellipsoid, measured by GNSS, and $\Phi, \Lambda$ are astronomical latitude and longitude provide by QDaedalus system from celestial equatorial coordinates $(\delta, \alpha)$ of measured stars (Völgyesi and Tóth 2015, 2016).

The main component of the QDaedalus system is an adapted Leica TCA 1800 robotic total station (Fig. 1). The eyepiece of the instrument is replaced with a CCD sensor. The GNSS receiver provides accurate timing of star observations and WGS84 coordinates. At the beginning of a measurement, important step is to calibrate the instrument. The calibration is made by a new solution using a special ATK2 collimator. The external computer controls the robotic total station via the QDaedalus software. Immediately after the measurement is completed, the software calculates the $\xi$ and $\eta$ components of DOV: 


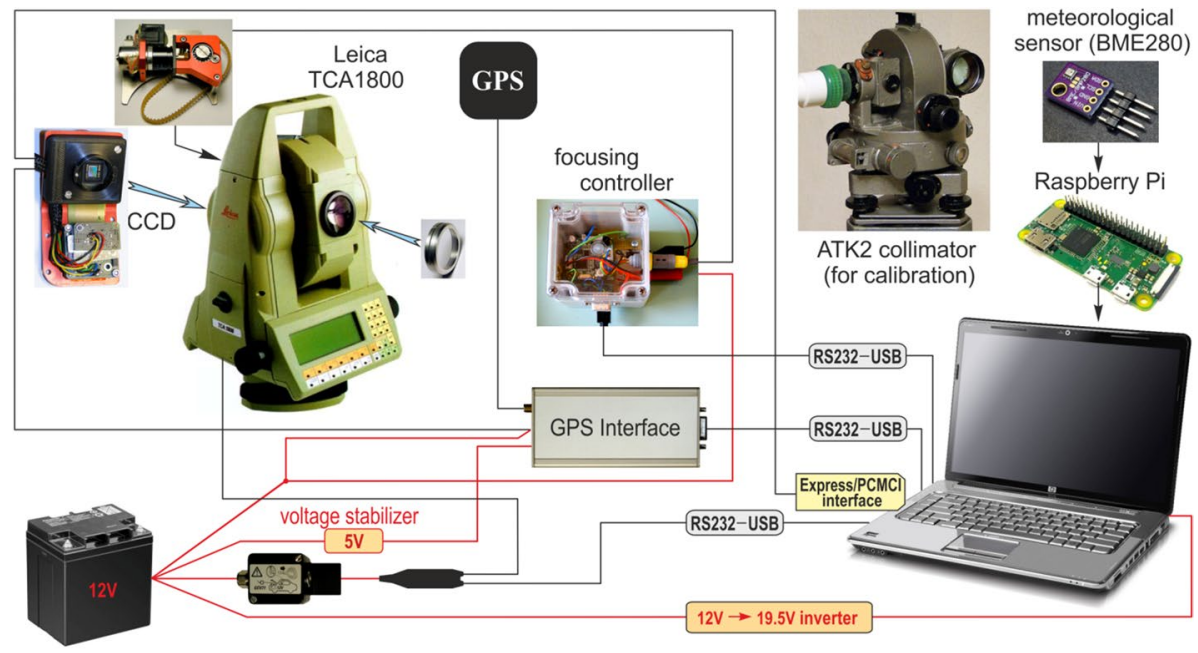

Fig. 1 Schematic construction of the QDaedalus system

\section{Measurements by QDaedalus system}

For the precise determination of DOV, a very long sequence was measured at the point Pistahegy. (the WGS84 coordinates of the measurement point are, $\varphi=47^{\circ} 24^{\prime} 53.8112^{\prime \prime}$ and $\lambda=19^{\circ} 08^{\prime} 17.8948^{\prime \prime}$ ) (Tóth and Völgyesi 2018). From the autumn of the year 2015 until now we performed 260 night measurements taken at this point in different seasons, in the most diverse meteorological and refraction conditions. On Fig. 2 the results of all 260 Qdaedalus measurements of DOV at the Pistahegy station can be seen, both the
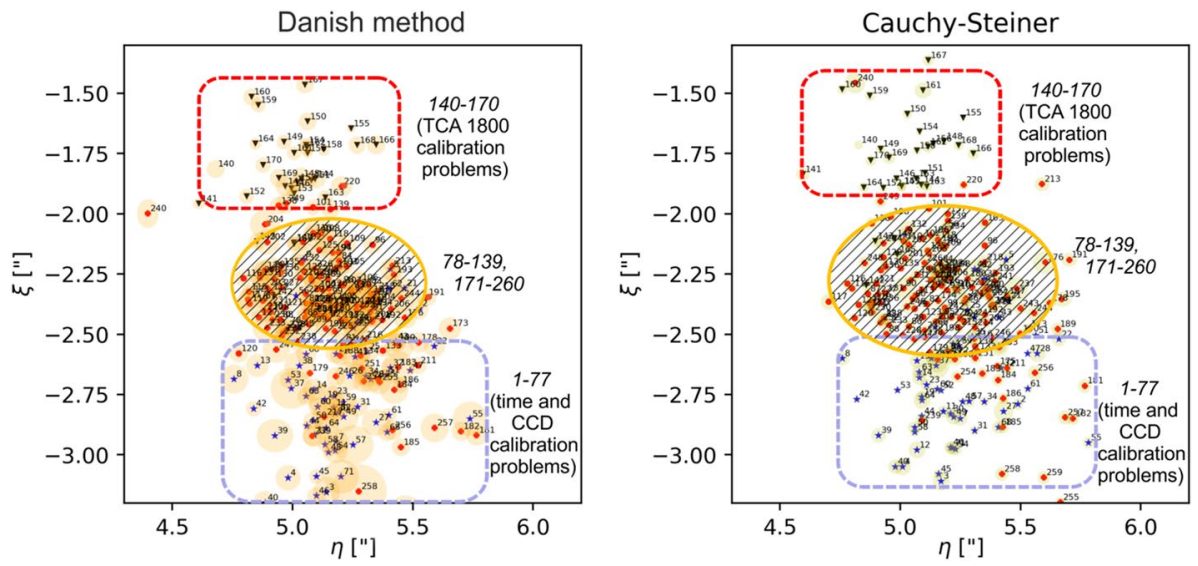

Fig. 2 DOV inversion results of 260 QDaedalus measurement series by the Danish method vs. CauchySteiner weights at Pistahegy Station. Numbers of series are given on the plots. Ellipses indicate estimated inversion errors. Median of $\xi$ is $-2.39^{\prime \prime} /-2.37 "$, median of $\eta$ is 5.15" /5.17" for Danish/Cauchy-Steiner methods, respectively 
results of the Danish method and the Cauchy-Steiner weights (Tóth and Völgyesi 2016) are presented.

Here we present a quick overview of the inversion procedure with Cauchy-Steiner weights. More details are found in Tóth and Völgyesi (2016). Robustification of the inversion with Cauchy-Steiner weights was successfully applied in other problems like e.g. Fourier Transformation (Szegedi and Dobróka 2014; Szegedi et al. 2014).

Our goal is to determine the model parameter vector

$$
\vec{m}=\left\{\Phi, \Lambda, \omega, i_{z}\right\}
$$

where $\Phi, \Lambda, \omega, i_{z}$ denote astronomical latitude, longitude, orientation correction, index error of the instrument, respectively. We have measurements for each epoch $i$ of the telescope axis and position of the star's image on the CCD sensor:

$$
\vec{d}_{m i}=\left\{\ell_{l}, z_{l}, x_{l}, y_{l}\right\}, \quad i=1,2, \ldots N
$$

where $N$ denotes the number of measurement epochs, $\ell_{i}, z_{i}, x_{i}, y_{i}$ denote direction of the telescope's pointing axis and position of the star image on the CCD, respectively. Model equations can be written for the $i$-th measurement epoch with calibration parameters $a_{11}$, $a_{12}, a_{21}, a_{22}, x_{0}, y_{0}$ as

$$
\begin{aligned}
& {\left[\ell_{i}-\ell_{i}^{*}(\Phi, \Lambda, \omega)\right] \sin z_{i}^{*}\left(\Phi, \Lambda, i_{z}\right)-a_{11}\left(x_{i}-x_{0}\right)-a_{12}\left(y_{i}-y_{0}\right)=0} \\
& z_{i}-z_{i}^{*}\left(\Phi, \Lambda, i_{z}\right)-a_{21}\left(x_{i}-x_{0}\right)-a_{22}\left(y_{i}-y_{0}\right)=0
\end{aligned}
$$

The general form of the above equations is $\vec{g}_{i}\left(\vec{d}_{e i}, \vec{m}\right)=0$, where $\vec{d}_{e i}=\left\{\ell_{l}, z_{l}, x_{l}, y_{l}\right\}$ denote theoretical values. The model equations are implicit in both data and parameters. The inversion solution for the parameter vector with Cauchy-Steiner weights is

$$
\vec{m}=\underline{\underline{G}}^{-g h} \vec{g}_{0}
$$

where the inverse matrix depends on the Cauchy-Steiner weights and the vector $g_{0}$ is the function value of $g$ at the Taylor point used in gradient linearization. The diagonal matrix $\underline{\underline{W^{\prime}}}$ of Cauchy-Steiner weights is

$$
\underline{\underline{W^{\prime}}}=\left\langle\ldots, \frac{\left(k \varepsilon^{\prime}\right)^{2}}{\left(k \varepsilon^{\prime}\right)^{2}+e_{i}^{\prime 2}}, \ldots\right\rangle
$$

and it depends on the residuals $e^{\prime}$, dihesion $\varepsilon^{\prime}$ and $k$ is the parameter of the $P_{k}$ product norm introduced by Steiner (1987).

At the start of measurements, the calibration of the CCD sensor and the inaccuracy of the computer clock caused various problems. Following the solution of the calibration and computer clock problem, after the first 77 measurements, the accuracy of QDaedalus measurements improved significantly. From the 140th measurement, another problem was realized in our measurements, the N-S components $\xi$ of DOV values began to increase more and more. We could not find the problem until the 170th measurement, and then it turned out that the parameters of the Leica TCA 1800 total station have been changed.

Repairing and calibrating the Leica TCA 1800 total station, after the 171th measurement the Qdaedalus system measures acceptable DOV values again. This experience underlies the need of regular instrument integrity check and calibration in case of high accuracy requirements. 
The results of problem-free measurement series 78 to 139 and 171 to 260 are located in hatched area in the middle of the Fig. 2, these points marked by red dots and the serial number of measurements are given next to the dots. The DOV values marked by blue stars computed from the first 77 measurements are separated in the lower part of the figure, and the values marked by black reverse triangles computed from the series of 140-170 Qdaedalus measurements can be found in the left upper part of the figure.

\section{Significance and measurement of meteorological parameters}

The biggest source of error in QDaedalus measurements is the atmospheric refraction. Atmospheric refraction is the deviation of light from a straight line as it passes through the atmosphere. Astronomical or celestial refraction causes stars to appear higher above the horizon than they actually are (see Fig. 3). This refraction is due to the velocity of light through air, decreasing with increased density. Air density is primarily a function of pressure, temperature and humidity.

So the amount of atmospheric refraction is a function of the temperature gradient, temperature, pressure, and humidity (the amount of water vapor, which is especially important at mid-infrared wavelengths). Refraction also depends on the color (wavelength) of the light. The degree of refraction also depends on the zenith angle and color of the stars. For example, in the visible spectrum, the shorter wavelength blue refracts more strongly than the longer wavelength red (see Fig. 3). Turbulent air can make distant objects appear to twinkle (atmospheric scintillation).

In previous measurements, meteorological parameters were determined only at the beginning and end of the measurements, but more recently we have switched to high-precision recording of barometric pressure, temperature, and humidity every $10 \mathrm{~s}$.

Meteorological parameters were measured with BME280 which is as a combined digital humidity, pressure and temperature sensor. The sensor module is housed in an extremely compact metal-lid LGA package with a footprint of only $2.5 \times 2.5 \mathrm{~mm}^{2}$ with a height of $0.93 \mathrm{~mm}$. At the heart of the module is the next-generation digital temperature, humidity and pressure sensor manufactured by Bosch-BME280. This precision sensor can measure relative humidity from 0 to $100 \%$ with $\pm 3 \%$ accuracy, barometric pressure from $300 \mathrm{~Pa}$ to $1100 \mathrm{hPa}$ with $\pm 1 \mathrm{hPa}$ absolute accuracy, and temperature from $-40{ }^{\circ} \mathrm{C}$ to $+85{ }^{\circ} \mathrm{C}$ with $\pm 1.0^{\circ} \mathrm{C}$ accuracy. This is available on a small module

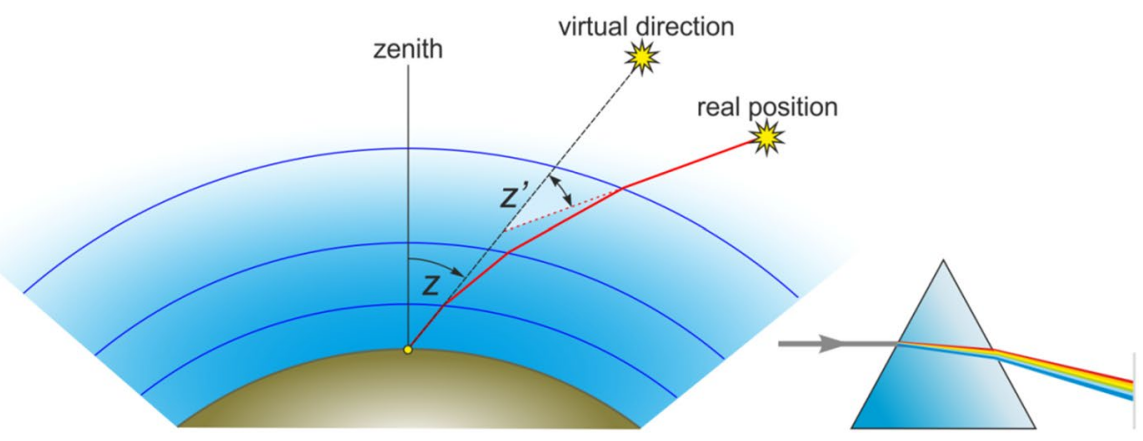

Fig. 3 Atmospheric refraction 


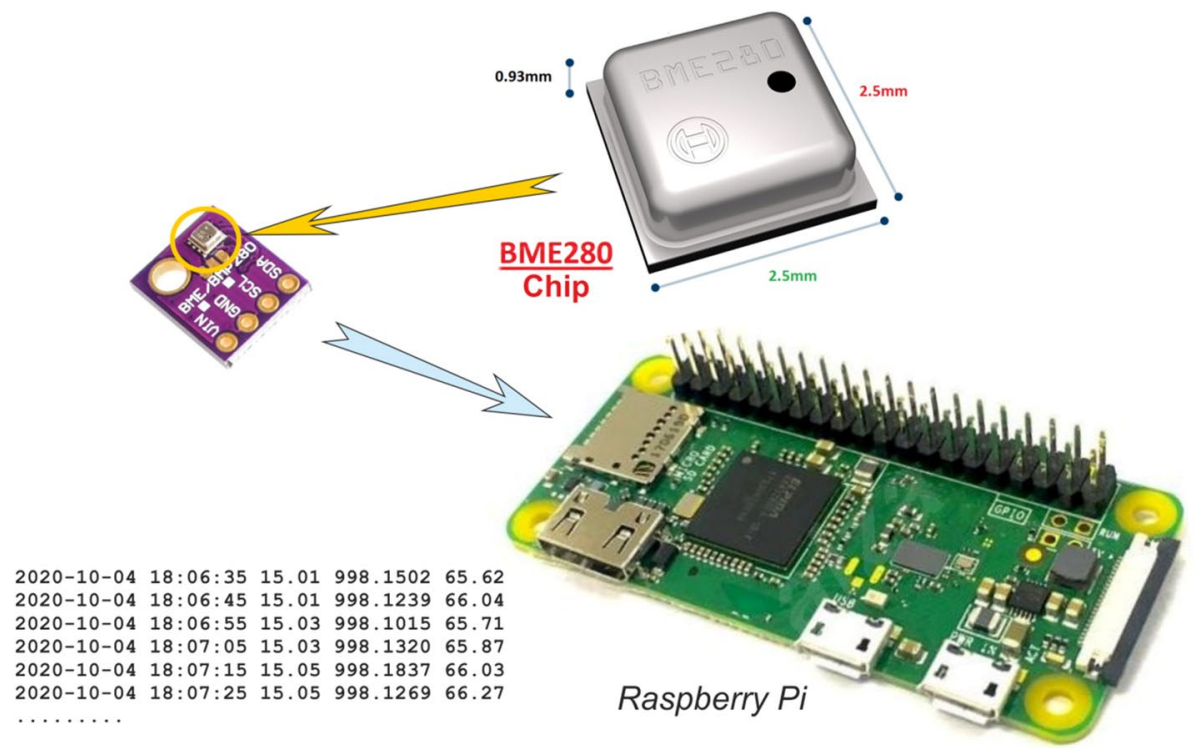

Fig. 4 Atmospheric refraction

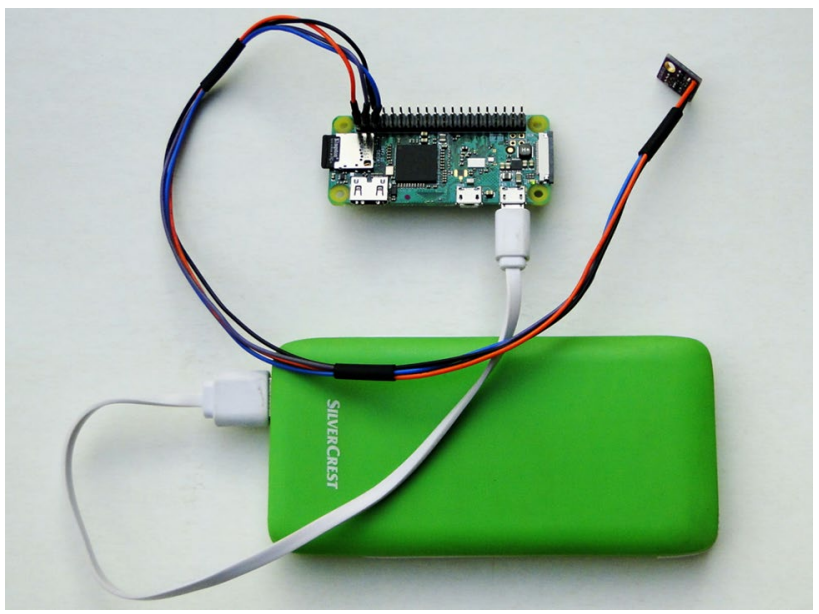

Fig. 5 Connection of BME280 to the Raspberry Pi

which provides access to the sensor via the I2C interface. BME280 is designed to be compatible with Raspberry $\mathrm{Pi}$, which can be programmed in python when connecting them. Figure 4 shows the Raspberry Pi and the compatible BME280 digital measuring unit. In the lower left part of the figure the format of the measured database can be seen (date, time, temperature, pressure and humidity). An easiest way to connect BME280 to the Raspberry Pi is shown in Fig. 5. 


\section{Improved modelling of radial-symmetric refraction for QDaedalus measurements}

Ground-based astrometrical observations at visible wavelengths are all affected by refraction. Astronomical refraction may be subdivided into a radial-symmetric and an asymmetric component, which is often called anomalous refraction.

Anomalous refraction is considered to be a major limiting factor for ground-based astrometry in general and astrogeodetic observations in particular (Hirt, 2006). While a digital zenith camera system is affected mostly by anomalous refraction close to the zenith, the QDaedalus system is affected by both anomalous and radial-symmetric parts. Therefore it is essential to model the latter, radial-symmetric part accurately.

In the following we describe an improved modeling of radial-symmetric refraction (which will be called simply refraction hereafter) with respect to our previous simple model. This improved modeling consists of two parts. First, refraction strongly depends on the local meteorological parameters at the observation site. Therefore continuous precise monitoring of these parameters has been implemented which facilitates more accurate, time-dependent calculation of refraction. Second, refraction also depends on the wavelength of the observed light. Measured stars have different spectral characteristics, and the value of refraction should vary with the spectral type depending on how the spectrum is distributed across the instrument's pass band (Stone, 1996).

\section{Refraction calculated from in-situ monitoring of meteorological parameters}

Due to (radial-symmetric) refraction apparent zenith distance $z^{\prime}$ of a star will be less than its true topocentric zenith distance $z$ by the amount of refraction $r$. Our previous simple refraction model employed Meeus' (1998) formula (16.1) with temperature $T$ in ${ }^{\circ} \mathrm{C}$ and air pressure $p$ in $\mathrm{hPa}$ :

$$
r(T, p)=\frac{p}{1010} \frac{283}{273+T}\left(c_{1} \tan z^{\prime}-c_{2} \tan ^{3} z^{\prime}\right)
$$

where $\mathrm{c}_{1}=58.294^{\prime \prime}$ and $\mathrm{c}_{2}=0.0668^{\prime \prime}$. In this model we used mean temperature and pressure calculated over the measurement period.

With a continuous in-situ monitoring of temperature, pressure and relative humidity during a measurement session, time-varying refraction can be calculated with formula (8). We logged these data with $10 \mathrm{~s}$ sampling rate and linearly interpolated both temperature and pressure to each measurement epoch. Figure 6 shows the logged environmental data for a particular measurement at the Pistahegy site.

During the 30 min measurement session temperature fluctuates by $1{ }^{\circ} \mathrm{C}$ and pressure increases by $0.3 \mathrm{hPa}$. Variation of refraction with respect to mean temperature and pressure amounts to $\pm 0.06^{\prime \prime}$ according to Fig. 7 . All the measured zenith angles were in the range of $30^{\circ} \pm 2^{\circ}$.

If we take into account that the standard deviation of vertical deflection components obtained for this particular measurement is about 0.05" with Cauchy-Steiner weights (Tóth and Völgyesi 2016), it is obvious that variation of meteorological parameters should be included in refraction modeling whenever possible. The average bias 

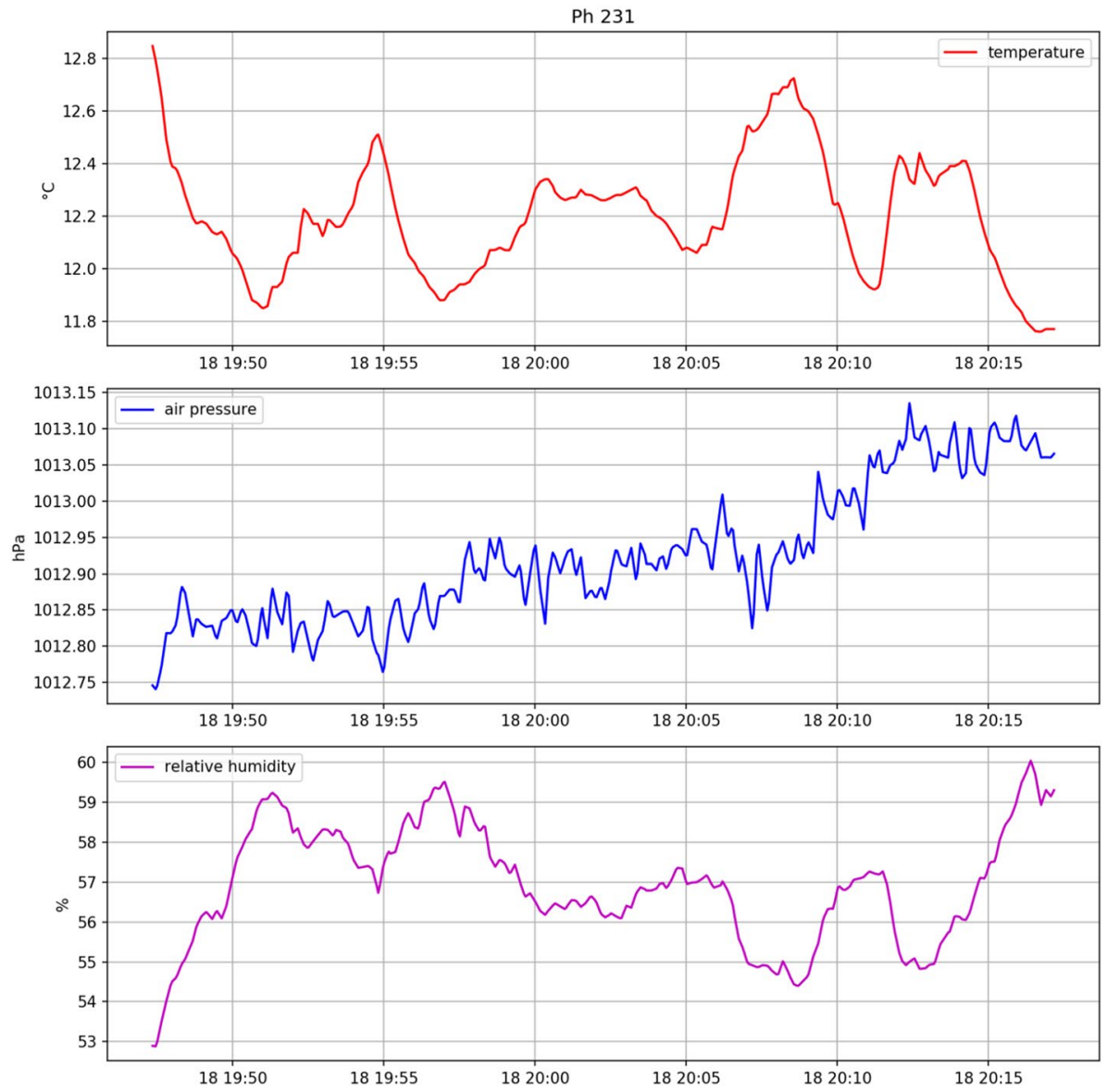

Fig. 6 Temperature, pressure and relative humidity at the Pistahegy site measured on 09.18.2020. Time axis labels show day and UTC time (hh:mm)

in estimated N-S and E-W vertical deflection components for 8 measurements (each 30 min long) is below $0.006^{\prime \prime}$ and $0.002 "$, respectively, if we neglect variation of temperature and pressure (Fig. 8). The standard deviations of the differences, however, is $0.012^{\prime \prime}$ and $0.006 "$ for the N-S and E-W components.

When DOV components are calculated with $0.001 "$ precision, it is important that coordinates of the stars used for the processing should be defined with at least the same precision. We found, however, that in the original QDaedalus star database declinations and right ascensions of the stars were given only with precisions 0.01 " and $0.01 \mathrm{~s}$ $\left(0.15^{\prime \prime}\right)$ from the FK6 star catalogue (Wielen et al. 1999). Hence we updated the original star catalogue with more accurate FK6 star coordinates (precise at least to 0.00001" and $0.000001 \mathrm{~s}$ in declination and right ascension). It can be seen in Fig. 9 that rounding of star coordinates affected the DOV components significantly, because average bias of the E-W component was $0.055^{\prime \prime}$ using less precise coordinates. 
$\mathrm{Ph} 231$

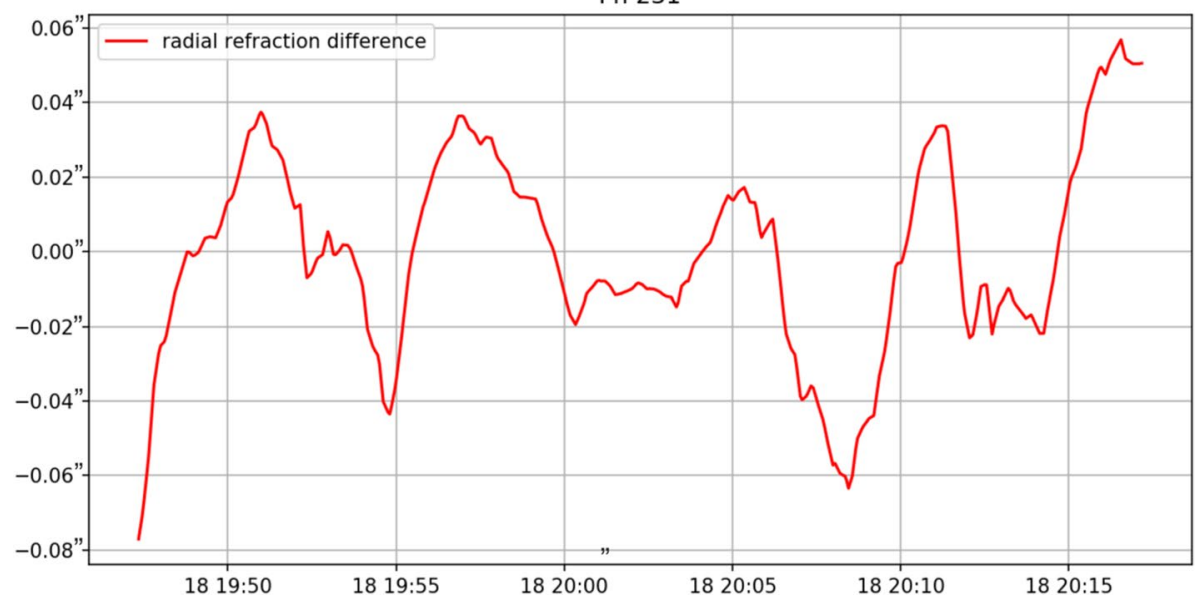

Fig. 7 Variation of the refraction angle in arcsec at the Pistahegy site with respect to average meteorological parameters on 09.18.2020. Time axis labels show day and UTC time (hh:mm)
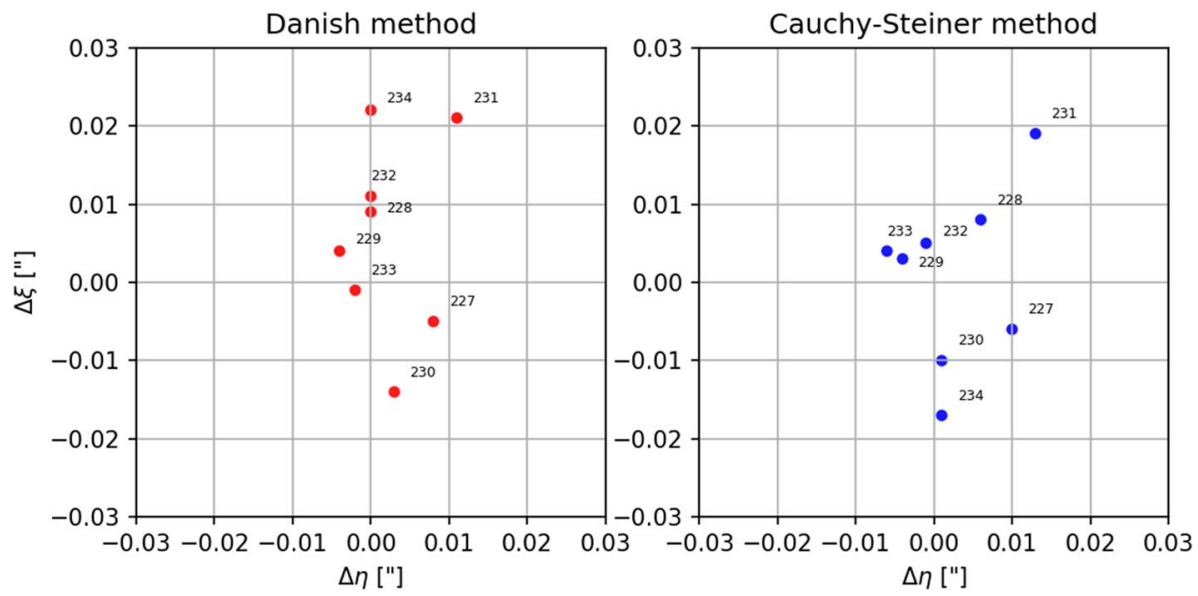

Fig. 8 Deflection of the vertical differences at Pistahegy site (measurements 227-234) resulted from processing with continuously monitored temperature and air pressure with respect to processing with average meteorological parameters, obtained with two standard processing methods

\section{Refraction calculations by taking into account star spectra}

Theoretically, refraction should be determined by tracing the path of light through the atmosphere. However, in lack of detailed knowledge of the atmospheric temperature, pressure and water vapor along this path, a more practical approach may be followed. This approach requires only knowledge of the meteorological conditions recorded at the observing site with each observation. This approach is very simple and fast and is also very accurate for zenith distances under $75^{\circ}$ (Smart 1965; Green 1985). The computation for refraction is reduced to a boundary-value problem with this formulation. Since refraction $r$ 


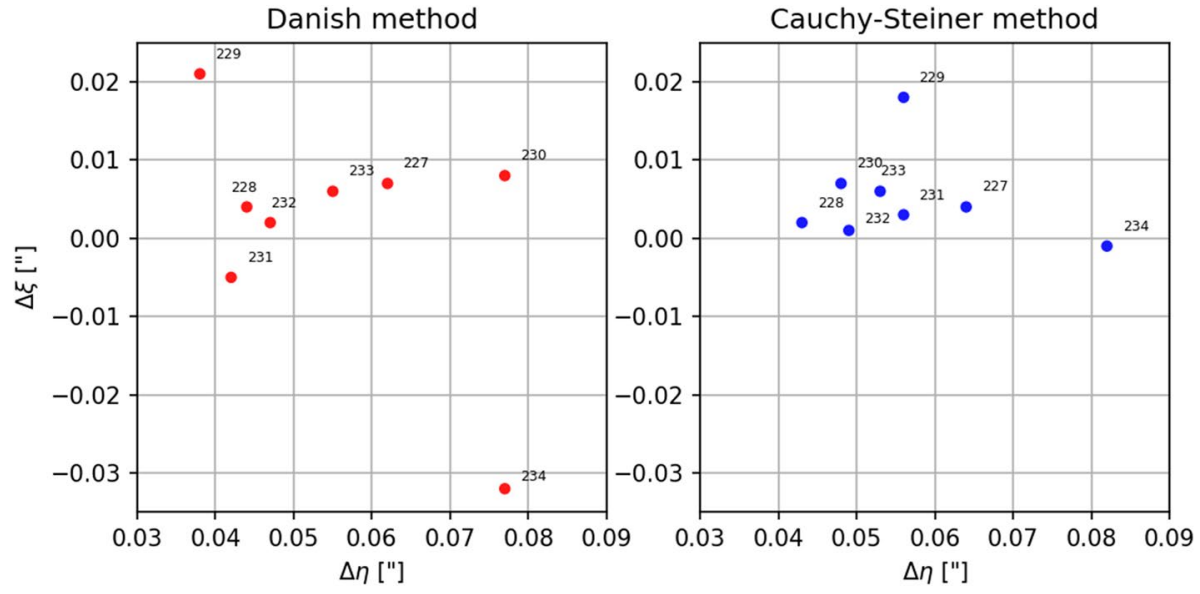

Fig. 9 Deflection of the vertical differences at Pistahegy site (measurements 227-234) resulted from processing with more precise star catalogue coordinates, obtained with two standard processing methods

depends on the wavelength of light $\lambda$, for accurate computation of the radial component of refraction this dependence must also be taken into account.

We first summarize the formulation for the calculation based on Stone (1996). According to the above theory, the refraction $r(\lambda)$ at the observing site can be computed by first computing the index of refraction of air $n_{0}$ at the site and then evaluating the expressions

$$
\begin{gathered}
\gamma=n_{0}-1 \\
\beta=0.001254\left(\frac{273.15+t}{273.15}\right) \\
\kappa=g_{0} / g=1+0.005302 \sin ^{2} \phi-0.00000583 \sin ^{2}(2 \phi)-0.000000315 h \\
r(\lambda)=\kappa \gamma(1-\beta) \tan z^{\prime}-\kappa \gamma(\beta-\gamma / 2) \tan ^{3} z^{\prime}
\end{gathered}
$$

where $t$ is known air temperature in ${ }^{\circ} \mathrm{C}$ at the site, $g$ is the sea-level gravity at the Earth's equator, $g_{0}$ is gravity at the observing site, $\phi$ is astronomical latitude of the observing site, $h$ is the height above the Earth's surface and $z^{\prime}$ is the apparent zenith distance of a star.

The index of refraction of air at wavelength $\lambda(\AA)$ can be computed accurately from known air temperature $T(\mathrm{~K})$, atmospheric pressure $P_{s}(\mathrm{hPa})$ of dry air (atmospheric pressure corrected for water vapor) and water vapor pressure $P_{w}(\mathrm{hPa})$ using the empirical approximations given by Owens (1967). According to Owens, the index of refraction of air $n_{0}(\lambda)$ can be computed very accurately using the equations given in Stone (1996). The water vapor pressure $P_{w}$ can be computed from the known ambient temperature $t\left({ }^{\circ} \mathrm{C}\right)$ and relative humidity $R H$ using the following formulas:

$$
x=\ln \frac{R H}{100}
$$




$$
\begin{gathered}
t_{d}=238.3\left[\frac{(t+238.3) x+17.2694 t}{(t+238.3)(17.2694-x)-17.2694 t}\right] \\
P_{w}=1.333224\left(4.50874+0.341724 t_{d}+0.0106778 t_{d}^{2}+0.184889 \cdot 10^{-3} t_{d}^{3}\right. \\
\left.+0.238294 \cdot 10^{-5} t_{d}^{4}+0.203447 \cdot 10^{-7} t_{d}^{5}\right)
\end{gathered}
$$

Next, using the absolute temperature $T=273.15+t, P_{s}=p-P_{w}$ and $\sigma=10^{3} / \lambda$ we calculate differential index of refraction from the formulas

$$
\begin{gathered}
D_{s}=\left[1+P_{s}\left(57.90 \cdot 10^{-8}-\frac{9.3250 \cdot 10^{-4}}{T}+\frac{0.25844}{T^{2}}\right)\right] \frac{P_{s}}{T} \\
D_{w}=\left[1+P_{w}\left(1+3.7 \cdot 10^{-4} P_{w}\right)\left(-2.37321 \cdot 10^{-3}+\frac{2.23366}{T}-\frac{710.792}{T^{2}}+\frac{7.75141 \cdot 10^{4}}{T^{3}}\right)\right] \frac{P_{w}}{T} \\
n_{0}-1=10^{-8}\left[2371.34+\frac{683939.7}{130-\sigma^{2}}+\frac{4547.3}{38.9-\sigma^{2}}\right] D_{s} \\
+\left(6487.31+58.058 \sigma^{2}-0.71150 \sigma^{4}+0.08851 \sigma^{6}\right) D_{w}
\end{gathered}
$$

The previously discussed refraction $r(\lambda)$ is only for monochromatic light of wavelength $\lambda$. In reality observations with QDaedalus are made through the optical pass band from about $350 \mathrm{~nm}$ to $750 \mathrm{~nm}$. As discussed in Stone (1984), a good approach is to calculate mean refraction $r_{m}$ by weighting the individual selective refractions $r(\lambda)$ with the apparent stellar flux at wavelength $\lambda$ and averaging across the pass band. The mean refraction is given then by

$$
r_{m}=\frac{\int_{0}^{\infty} S(\lambda) E(\lambda) A(\lambda) L(\lambda) D(\lambda) r(\lambda) d \lambda}{\int_{0}^{\infty} S(\lambda) E(\lambda) A(\lambda) L(\lambda) D(\lambda) d \lambda}
$$

where $S(\lambda)$ is the spectral energy distribution of the star being observed; $E(\lambda)$ is the transmittance of the interstellar dust; $A(\lambda)$ is the transmission of the atmosphere; $L(\lambda)$ is the transmission of the telescope optics; $D(\lambda)$ is the quantum efficiency of the detector being used and $r(\lambda)$ is the selective refraction.

The function $E(\lambda)$ in (19) is given by

$$
E(\lambda)=10^{-0.4 R E(B-V) a(\lambda)}
$$

where $a(\lambda)$ is the interstellar absorption magnitudes at wavelength $\lambda$, parameter $R$ is defined as the absorption ratio $(R \sim 3)$, and $E(B-V)$ is the color excess of the star being observed. The absorption has been normalized to a reddening $R E(B-V)=1.0$ and a numerical approximation is given as:

$$
a(\lambda)=-0.544472+\frac{0.995958}{\lambda}-\frac{0.0805414}{\lambda^{2}}
$$

In Eq. (21) wavelength $\lambda$ should be given in $\mu \mathrm{m}$. The atmospheric transmission $A(\lambda)$ at zenith distance $z$ is given by

$$
A(\lambda)=a^{\prime}(\lambda)^{\sec z^{\prime}}
$$


where

$$
a^{\prime}(\lambda)=1.33425-\frac{0.584170}{\lambda}+\frac{0.290928}{\lambda^{2}}-\frac{0.0676255}{\lambda^{3}}
$$

In Eq. (23) wavelength $\lambda$ should be given in $\mu \mathrm{m}$.

The spectral energy distribution $S(\lambda)$ of the star as well as the $E(B-V)$ color excess can be determined from the spectral type of the star. A comprehensive spectrophotometric library of stars can provide such an information. We have used the spectral library compiled by Jacoby et al. (1984), which contains spectra for 161 stars having spectral classes O-M and luminosity classes V, III and I. The spectra extend from 351 to $742.7 \mathrm{~nm}$ at a resolution of $\sim 0.45 \mathrm{~nm}$. An example plot of the radiative intensity of the HD 66,171 star from G2 spectral and V luminosity class from this library is shown in Fig. 10.

To reduce computation time we interpolated spectral data to $0.1 \mathrm{~nm}$ stepsize and then integrated stellar fluxes over $5 \mathrm{~nm}$ intervals. Finally we normalized the fluxes $S(\lambda)$ to unit magnitude. Integrated and normalized fluxes between 355 and $740 \mathrm{~nm}$ as well as $E(B-V)$ color excess data were then saved into an Sqlite database for fast access.

The QDaedalus system's CCD sensor is F-080 from Allied Vision that includes a Sony ICX-204 CCD detector. Monochrome quantum efficiency $D(\lambda)$ of this detector is shown in Fig. 11. These data were digitized from 400 to $740 \mathrm{~nm}$ in $5 \mathrm{~nm}$ steps and stored in a Sqlite database.

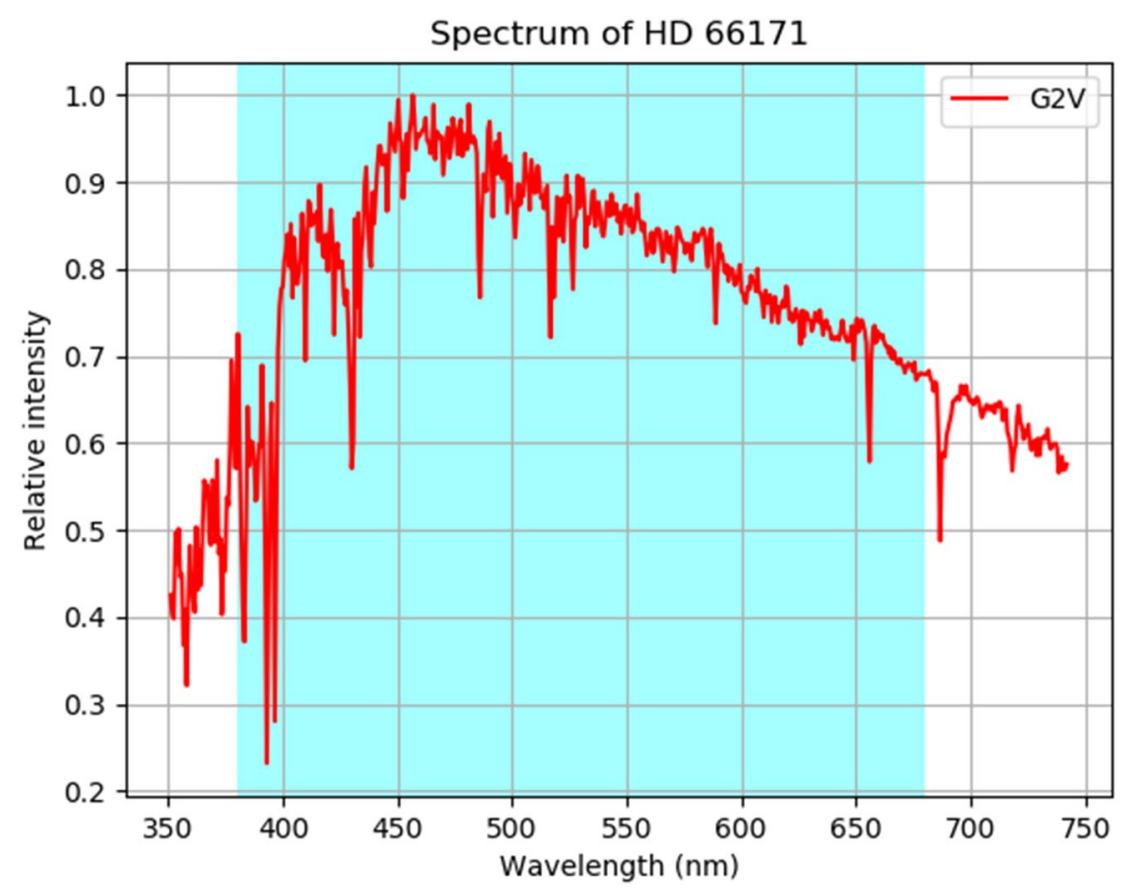

Fig. 10 Spectrum of the HD 66,171 star of G2 spectral and V luminosity class from the Jacoby library of stellar spectra (Jacoby et al. 1984) 


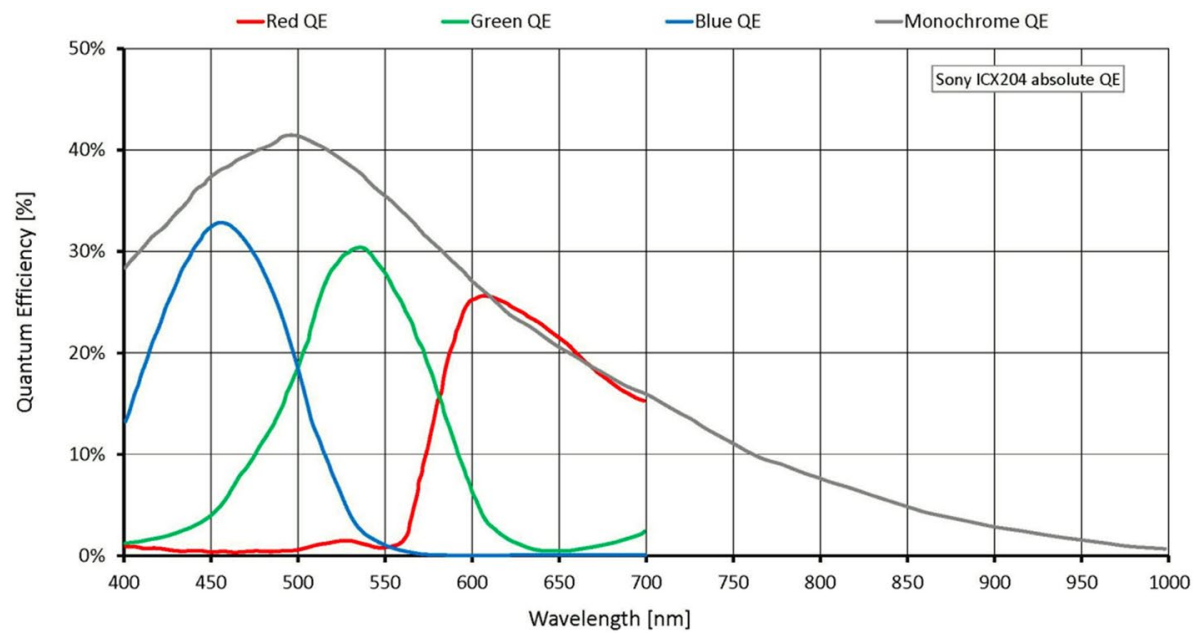

Fig. 11 Quantum efficiency of the Sony ICX-204 detector in the $400 \mathrm{~nm}-1000 \mathrm{~nm}$ spectral range (Allied Vision, Guppy F-080 DataSheet V5.2.2)

Transmission of the telescope optics $L(\lambda)$ was taken to be unity in the $400 \mathrm{~nm}-1000 \mathrm{~nm}$ spectral range, because optical glass materials have nearly unit transmissivity in this range (Edmund Optics 2021).

Since the original star catalogue of the QDaedalus system did not contain spectral and luminosity classes of the stars, we included these data from the Hipparcos catalogue (van Leeuwen, 2007) for all of the 4311 stars in the database.

Selective refraction $r(\lambda)$ is calculated for each epoch and each wavelength using the measured temperature, air pressure and humidity using Eq. (12). Calculation of mean refraction $r_{m}$ for a measured star is then performed via Eq. (19) by numerical integration with $5 \mathrm{~nm}$ stepsize in the 400-740 $\mathrm{nm}$ spectral range.

As discussed above, the value of refraction should vary with the spectral type depending on how the spectrum of a star is distributed across the pass band. Stone (1996) calculated differential color refraction for various instrumental pass bands with respect to an F-type star, and got values between $-0.115^{\prime \prime}$ and $0.101^{\prime \prime}$ for $45^{\circ}$ zenith distance.

We calculated refraction using environmental data with and without taking into account the spectra of measured stars. Utilizing the above algorithm for QDaedalus measurements we calculated refraction differences for stars measured near zenith distance $30^{\circ}$ that resulted from using spectral information in the computation. Figure 12 shows an example plot of these differences for a particular standard $30 \mathrm{~min}$ measurement. As we see the differences are in the range $-0.125^{\prime \prime} \div 0.070^{\prime \prime}$, in agreement with Stone (1996).

It is obvious that variation of refraction due to the different colors of stars (spectra) should be included in refraction modeling. The average biases in estimated N-S and E-W vertical deflection components for 34 measurements were -0.009 " and $-0.003^{\prime \prime}$, respectively, if we neglect stellar spectra (Fig. 13). The standard deviations of differences are $0.013 "$ and $0.018 "$ for the N-S and E-W components. 


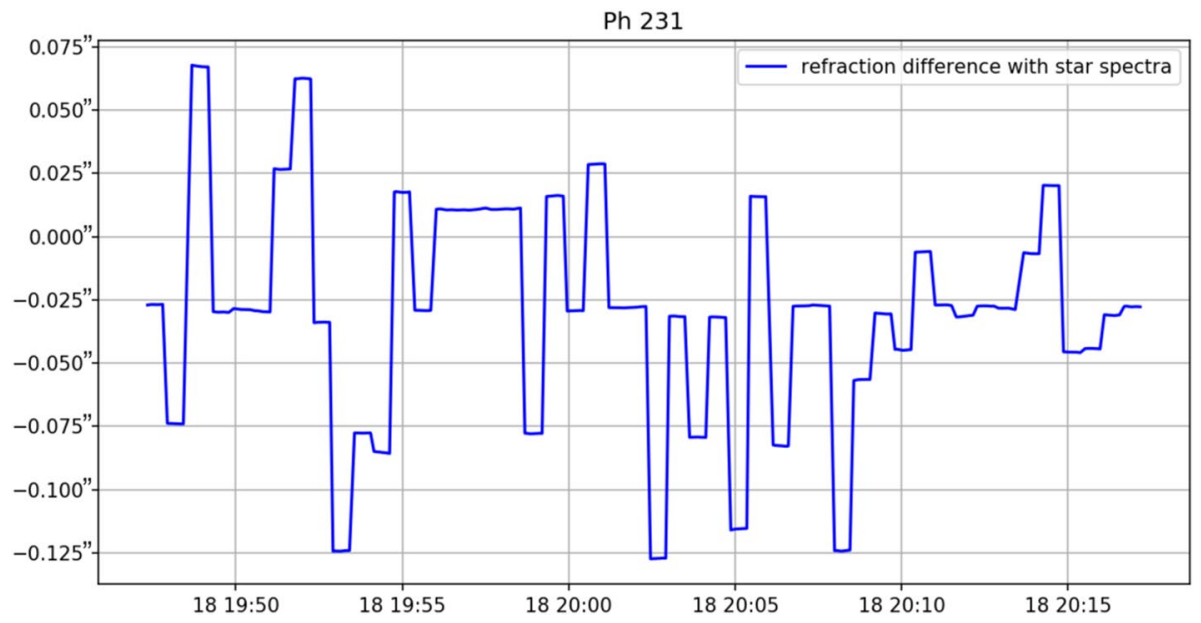

Fig. 12 Variation of the refraction angle in arc sec at the Pistahegy site by taking into account colors of stars. Standard 30 min measurement No. 231 on 09.18.2020 is shown. Time axis labels show day and UTC time (dd hh:mm)
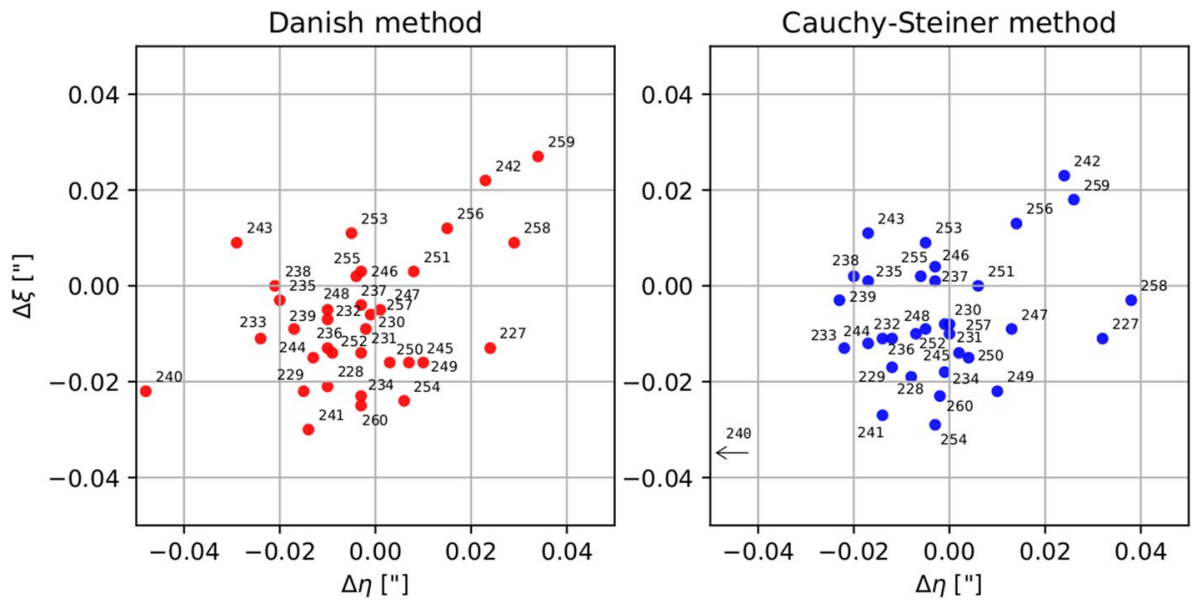

Fig. 13 Deflection of the vertical differences at Pistahegy site (34 measurements No. 227-260) resulted from processing with star spectra with respect to processing without it, obtained with two standard processing methods. Continuously monitored environmental parameters were taken into account in both cases

\section{Conclusion}

Astronomical deflections of the vertical measured by the QDaedalus system are significantly influenced by atmospheric refraction, which depends primarily on the environmental parameters (air pressure, temperature, humidity) and the spectrum of the stars.

In the last 7 years we performed 260 measurements taken at Pistahegy point at the southern part of Budapest in different seasons, in the most diverse meteorological, temperature and refraction conditions. In previous measurements, meteorological parameters 
were determined only at the beginning and end of the measurements, but more recently we have switched to high-precision recording of barometric pressure, temperature, and humidity every $10 \mathrm{~s}$. This made it possible to continuously determine the refraction and take its effect into account during the entire measurement time. During the 30 min measurements time session temperature fluctuated by $1{ }^{\circ} \mathrm{C}$ and pressure changed by $0.3 \mathrm{hPa}$. All the measured zenith angles were in the range of $30^{\circ} \pm 2^{\circ}$. Compared to the value calculated for the average temperature and air pressure, the refraction changed by \pm 0.06 ".

We want to emphasize, that although we applied two standard inversion procedures with Danish and Cauchy-Steiner weights, the latter gave consistently better results in terms of both accuracy of the inversion parameters and correlation norm. This is probably due to the higher statistical efficiency of M-inversion in cases of non-Gaussian distribution of residuals compared with the robust E-estimation with Danish method commonly used in geodetic data processing.

Refraction also depends on the wavelength of the observed light. Measured stars have different spectral characteristics, and the value of refraction should vary with the spectral type depending on how the spectrum is distributed across the instrument's pass band. We calculated refraction using environmental data with and without taking into account the spectra of measured stars. Utilizing our algorithm for QDaedalus measurements we calculated refraction differences for stars measured near zenith distance $30^{\circ}$ that resulted from using spectral information in the computation. The differences were in the range $-0.125^{\prime \prime} \div 0.070^{\prime \prime}$. It is obvious that variation of refraction due to the different colors of stars (spectra) should be included in refraction modeling. The average biases in estimated N-S and E-W vertical deflection components for 34 measurements were $-0.009^{\prime \prime}$ and $-0.003^{\prime \prime}$, respectively, if we neglect stellar spectra. The standard deviations of differences are $0.013^{\prime \prime}$ and $0.018 "$ for the N-S and E-W components.

Acknowledgements Our investigations was supported by the National Scientific Research Fund, Project numbers OTKA K-124286.

Author contributions Lajos Völgyesi: conceptualization, measurements, review and editing, visualization. Gyula Tóth: conceptualization, methodology, software, visualization.

Funding Open access funding provided by Budapest University of Technology and Economics. The research was carried out in the project No. K-124286 supported by the National Research, Development and Innovation Office (NKFIH).

Avilability of data and material Because of the data confidentiality, the experimental data is not published.

Code availability Because of the data confidentiality, the code is not published.

\section{Declarations}

Conflicts of interest The authors have no conflicts of interest to declare that are relevant to the content of this article.

Open Access This article is licensed under a Creative Commons Attribution 4.0 International License, which permits use, sharing, adaptation, distribution and reproduction in any medium or format, as long as you give appropriate credit to the original author(s) and the source, provide a link to the Creative Commons licence, and indicate if changes were made. The images or other third party material in this article are included in the article's Creative Commons licence, unless indicated otherwise in a credit line to the material. If material is not included in the article's Creative Commons licence and your intended use is not permitted by statutory regulation or exceeds the permitted use, you will need to obtain permission directly from the copyright holder. To view a copy of this licence, visit http://creativecommons.org/licenses/by/4.0/. 


\section{References}

Bürki B, Guillaume S, Sorber P, Oesch PH (2010) Daedalus A Versatile Usable Digital Clip-on Measuring System for Total Stations. International Conference on Indoor Positioning and Indoor Navigation (IPIN). IEEE, Zürich, Switzerland. 15-17

Edmund Optics (2021) Website https://www.edmundoptics.com/knowledge-center/application-notes/optics/ optical-glass/ (last visited: 21.03.2021.)

Green RM (1985) Spherical Astronomy. Cambridge University Press, Cambridge

Hauk M, Hirt C, Ackermann C (2016) Experiences with the QDaedalus system for astrogeodetic determination of deflections of the vertical. Surv Rev 49(355):294-301. https://doi.org/10.1080/00396265.2016. 1171960

Hirt C (2006) Monitoring and analysis of anomalous refraction using a digital zenith camera system. Astron Astrophys 459(1):283-290

Hirt C, Bürki B, Somieski A, Seeber G (2010) Modern determination of vertical deflections using digital zenith cameras. J Surv Eng 136(1):1-12. https://doi.org/10.1061/_ASCE_SU.1943-5428.0000009

Jacoby GH, Hunter DA, Christian CA (1984) A library of stellar spectra. Astrophys J Suppl Ser 56:257-281

Meeus J (1998) Astronomical algorithms. 2nd Edition, Willmann-Bell, Richmond, Virginia, USA

Owens JC (1967) Optical Refractive Index of Air: Dependence on Pressure, Temperature and Composition. Appl Opt 6:51-59

Smart WM (1965) Spherical Astronomy. Cambridge University Press, Cambridge

Steiner F (1987) Possibilities to realize higher efficiency in geophysical interpretation. Geophysical Transactions 33(1):3-9

Stone RC (1984) The effect of differential color refraction on declinations determined in meridian circle programs. Astron Astrophys 138:275-284

Stone RC (1996) An accurate method for Computing Atmospheric Refraction. Publ Astron Soc Pac 108:1051-1058

Szegedi H, Dobróka M (2014) On the use of Steiner's weights in inversion-based Fourier transformation: robustification of a previously published algorithm. Acta Geod Geophys 49:95-104. https://doi.org/10. 1007/s40328-014-0041-0

Szegedi, H., Dobroka, M., \& Molnar, J. S. (2014). A New Robust Inversion Method Using Cauchy-Steiner Weights-And Its Application in Data Processing. In Near Surface Geoscience 2014-20th European Meeting of Environmental and Engineering Geophysics. European Association of Geoscientists \& Engineers, Netherland. https://doi.org/10.3997/2214-4609.20142078.

Tóth Gy, Völgyesi L (2016) Data processing of QDaedalus measurements. Geosciences and Engineering 5(8):149-166

Tóth Gy, Völgyesi L (2018) Experiences of QDaedalus measurements. Geosciences and Engineering 6(9):75-86

van Leeuwen F (2007) Validation of the new Hipparcos reduction. Astron Astrophys 474:653-664

Völgyesi L, Tóth G (2016) Possibilities of geodetic application of the QDaedalus system, Geodézia és Kartográfia, LXVIII: (9-10), 11-17. (In Hungarian)

Völgyesi L, Tóth G (2015) Possibilities of determinations of the vertical deflections. Geomatikai Közlemények, XVIII 2:75-83 (In Hungarian)

Wielen R, Schwan H, Dettbarn C, Lenhardt H, Jahreiss H \& Jährling R (1999) Sixth Catalogue of Fundamental Stars (FK6). Part I. Basic fundamental stars with direct solutions. Veroeffentlichungen des Astronomischen Rechen-Instituts Heidelberg, 35, 1. 